\title{
An optimal linear solver for the Jacobian system of the extreme type-II Ginzburg-Landau problem
}

\author{
N. Schlömer ${ }^{\mathrm{a}}, \mathrm{W}$. Vanroose ${ }^{\mathrm{a}}$ \\ ${ }^{a}$ Universiteit Antwerpen, Departement Wiskunde-Informatica, Middelheimlaan 1 , \\ 2020 Antwerp, Belgium
}

\begin{abstract}
This paper considers the extreme type-II Ginzburg-Landau equations, a nonlinear PDE model for describing the states of a wide range of superconductors. Based on properties of the Jacobian operator and an AMG strategy, a preconditioned Newton-Krylov method is constructed. After a finite-volume-type discretization, numerical experiments are done for representative two- and three-dimensional domains. Strong numerical evidence is provided that the number of Krylov iterations is independent of the dimension $n$ of the solution space, yielding an overall solver complexity of $O(n)$.
\end{abstract}

Keywords: Ginzburg-Landau equations, preconditioning, algebraic multigrid

\section{Introduction}

The nonlinear Schrödinger equation is used in many areas of science and technology and describes, for example, the propagation of solutions in fiber optics [1] and Bose-Einstein condensates in ultra-cold traps [2]. Prototypical for this type of models is the Ginzburg-Landau problem, widely used to study the state of both low- and high-temperature superconductors. Due to its highly nonlinear nature, the involved energy landscape, and the strong dependence of solutions on external conditions, numerical simulations of the Ginzburg-Landau model have become an essential tool for providing better insight into properties of superconductivity phenomena.

The Ginzburg-Landau model has attracted wide interest since its inception in the 1950s. In particular, the work on the linearization by Abrikosov of the model around the upper critical field is widely known 3. The mathematical foundations for the equilibrium Ginzburg-Landau models are well developed 4, 5 and a framework for finite element and finite volume discretizations was provided [6]. Different types of discretizations and numerical approximations of the Ginzburg-Landau models have been developed since, all of which subject to numerical simulations.

Throughout the physics literature, several methods for solving the GinzburgLandau equations are described. Used most prominently is a Gauss-Seidel-type iterative scheme [7, 8 that is readily implemented, yet fails to converge for systems with physically unstable vortex configurations. Furthermore, it only yields linear convergence close to a solution. In computational physics in general, the use of Newton-Krylov methods and nonlinear multigrid schemes such as FAS is widespread [9. Also, preconditioned Newton-Krylov methods are already 

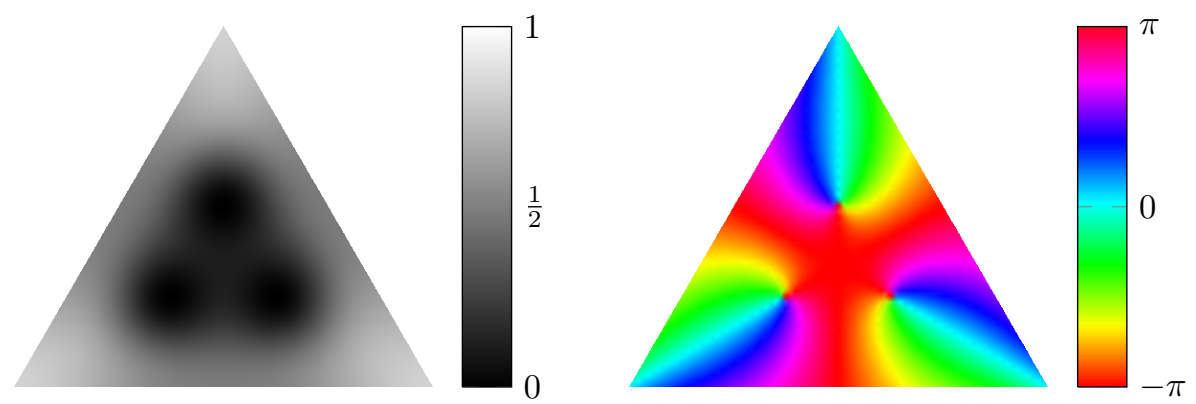

Figure 1: Typical solution $\psi: \Omega \mapsto \mathbb{C}$ (displayed as $|\psi|^{2}$, $\arg \psi$ ) of the extremetype-II Ginzburg-Landau equations, (2), here for a flat triangular domain with circumradius 5 and the magnetic vector potential $\boldsymbol{A}(x, y)=(-y / 2, x / 2)^{\mathrm{T}}$. Three of the characteristic vortices appear.

applied in other phase field models such as the Cahn-Hilliard equation [10]. Initial efforts to apply Newton-Krylov to the Ginzburg-Landau problem were taken in 11; preconditioning is not discussed though.

An important research topic in the context of the Ginzburg-Landau equations is the formation of vortex patterns in the solutions (see figures $1,6 \mathrm{c}, 6 \mathrm{~d}$ ). To understand the formation and dynamics of those patterns, the tools of nonlinear systems analysis can be employed. For example, numerical continuation techniques help computing a family of solutions as a function of a problem parameter, e.g., the strength of the externally applied magnetic field or the electric current at one of the boundaries. The main application of numerical parameter continuation is the construction of a bifurcation diagram that identifies the stability regions and the transition between stable and unstable patterns marked by bifurcation points [12. A systematic bifurcation analysis of the patterns that appear in mesoscopic superconductors is carried out for squareshaped domains in 13 . The main computational load in numerical continuation are the linear solves with the Jacobian operator. By the sheer number of unknowns, this is particularly expensive for discretizations of three-dimensional domains. It is thus required to develop linear solvers for which the memory requirements and the computational cost grows slowly with the number of unknowns. To the knowledge of the authors, no linear scalable method for the Ginzburg-Landau problem has been developed. It is the goal of this paper to display that an AMG-preconditioned Newton-Krylov method is a viable approach for the extreme-type-II Ginzburg-Landau equations.

The remainder of the paper is organized as follows. Section 2 reviews the Ginzburg-Landau equations for extreme-type-II superconductors; section 2.1 is concerned with its linearization, the Jacobian, and discusses properties with respect to numerical algorithms. While section 3 introduces the applied discretization and shows that many important properties carry over from the continuous framework, section 4 is concerned with the solution of the Jacobian system and introduces a multigrid strategy. The convergence behavior is explored through numerical experiments on representative two- and three-dimensional domains. The document concludes with a discussion of the obtained results. 


\section{The Gibbs energy and the continuous Ginzburg-Landau problem}

For an open, bounded domain $\Omega \subset \mathbb{R}^{3}$ with a piecewise smooth boundary $\partial \Omega$, the Ginzburg-Landau problem is usually stated as a minimization problem of the Gibbs energy functional

$$
\begin{aligned}
G(\psi, \boldsymbol{A})-G_{\mathrm{n}}=\xi \frac{|\alpha|^{2}}{\beta} \int_{\Omega}[ & -|\psi|^{2}+\frac{1}{2}|\psi|^{4}+\|-\mathrm{i} \boldsymbol{\nabla} \psi-\boldsymbol{A} \psi\|^{2} \\
& \left.+\kappa^{2}(\boldsymbol{\nabla} \times \boldsymbol{A})^{2}-2 \kappa^{2}(\boldsymbol{\nabla} \times \boldsymbol{A}) \cdot \boldsymbol{H}_{0}\right] \mathrm{d} \Omega
\end{aligned}
$$

over $\psi \in H_{\mathbb{C}}^{2}(\Omega)$ and $\boldsymbol{A} \in H_{\mathbb{R}^{n}}^{2}(\Omega)[6$. The scalar-valued function $\psi$ is commonly referred to as order parameter, $\boldsymbol{A}$ is the magnetic vector potential corresponding to the total magnetic field. The physical observables associated with the state $(\psi, \boldsymbol{A})$ are the density $\rho_{\mathrm{C}}=|\psi|^{2}$ of the superconducting charge carriers (Cooper pairs) and the magnetic field $\boldsymbol{B}=\boldsymbol{\nabla} \times \boldsymbol{A}$. The constant $G_{\mathrm{n}}$ represents the energy associated with the entirely normal (non-superconducting) state.

The energy (1) is presented in its dimensionless form, and it depends upon the impinging magnetic field $\boldsymbol{H}_{0}$ and the material parameters $\alpha, \beta, \lambda, \xi \in \mathbb{R}$. The ratio $\kappa:=\lambda / \xi$ of the penetration depth $\lambda$ (the length scale at which the magnetic field penetrates the sample) and the coherence length $\xi$ (the characteristic spatial scale of $\psi$ ) determines the type of the superconductor: It is said to be of type $I$ if $\kappa<1 / \sqrt{2}$, and of type II otherwise. The two types behave fundamentally differently when exposed to a magnetic field: Type I superconductors exhibit alternating superconducting and nonsuperconducting regions, while type II superconductors show vortex patterns [14] (see figure 1).

Starting from the Gibbs energy and using standard calculus of variations, it is possible to derive the Ginzburg-Landau equations 15, a boundary-value problem in the unknowns $\psi$ and $\boldsymbol{A}$. As anticipated in the introduction, we will simplify the problem and consider only the limit $\kappa \rightarrow \infty$ (extreme type-II superconductors): this approximation gives satisfactory results for all hightemperature superconductors which have large values of $\kappa$ (typically $50<\kappa$ ) In this case, the Ginzburg-Landau equations decouple for $\psi$ and $\boldsymbol{A}$, such that the magnetic vector potential $\boldsymbol{A}$ is given up to gauging by the applied magnetic field $\boldsymbol{H}_{0}$ through

$$
\boldsymbol{\nabla} \times \boldsymbol{A}=\boldsymbol{H}_{0} \quad \text { in } \mathbb{R}^{3},
$$

and $\psi \in X, X \subseteq H_{\mathbb{C}}^{2}(\Omega)$, by

$$
0=\mathcal{G L}(\psi):=\left\{\begin{array}{l}
(-\mathrm{i} \boldsymbol{\nabla}-\boldsymbol{A})^{2} \psi-\psi\left(1-|\psi|^{2}\right) \quad \text { in } \Omega \\
\mathbf{n} \cdot(-\mathrm{i} \boldsymbol{\nabla}-\boldsymbol{A}) \psi \quad \text { on } \partial \Omega
\end{array}\right.
$$

with $\mathbf{n}$ being the outer normal on $\partial \Omega$ and $X:=\left\{\psi \in H_{\mathbb{C}}^{2}(\Omega): \mathcal{G L}(\psi, \boldsymbol{A})\right.$ bounded $\}$, i.e., the natural energy space of (1).

As the domain is given in units of $\xi=\lambda / \kappa$, the large- $\kappa$ limit implies $\lambda \gg \xi$ which means that $\boldsymbol{H}_{0}$ is not disturbed by the magnetic field induced by the electric charge density $\rho_{C}$.

Note that, for any given $\chi \in \mathbb{R}$,

$$
\mathcal{G L}(\exp (\mathrm{i} \chi) \psi)=\exp (\mathrm{i} \chi) \mathcal{G L}(\psi) .
$$


Consequently, any given solution $\psi$ of the Ginzburg-Landau problem is really just a representative of a whole set of solutions $[\psi]:=\{\exp (\mathrm{i} \chi) \psi: \chi \in \mathbb{R}\}$. This expresses the fact that for superconducting states, the actual value of the argument of $\psi$ is of no physical relevance: $|\psi|^{2}$ represents the observable. As the complex argument of any coefficient does not play any role in the scalar multiplication, $c[\psi]=|c|[\psi], c \in \mathbb{C}, \psi \in X$, it is natural to restrict the scalar field to $\mathbb{R}$. The inner product in the vector space $X$ over the field $\mathbb{R}$ is

$$
\langle\phi, \psi\rangle_{\mathbb{R}}:=\Re\langle\phi, \psi\rangle=\Re\left(\int_{\Omega} \bar{\phi} \psi\right) .
$$

\subsection{The Jacobian, the kinetic energy operator, and their properties}

Equation (2) is a nonlinear equation in $\psi$ and hence classically suited for treatment with Newton's method. While there were efforts to solve (2) with a modified algorithm [16], the generic approach of the full Newton system is applied here for its attractive second-order convergence. In this section, properties of the (continuous) Jacobian system

$$
J(\psi) \delta \psi=-\mathcal{G L}(\psi)
$$

with

$$
J(\psi) \varphi:=\left((-\mathrm{i} \boldsymbol{\nabla}-\boldsymbol{A})^{2}-1+2|\psi|^{2}\right) \varphi+\psi^{2} \bar{\varphi} .
$$

will be discussed. Note that $J(\psi)$ is only linear if $X$ is defined as vector space over the field $\mathbb{R}$.

The kinetic energy operator. Before analyzing the Jacobian operator $J(\psi)$ as a whole, we will take a close look at the part that is commonly referred to as the kinetic energy operator,

$$
K \varphi:=(-\mathrm{i} \boldsymbol{\nabla}-\boldsymbol{A})^{2} \varphi .
$$

This operator is linear in $X$ and self-adjoint with respect to the ordinary $L^{2}(\Omega)$ inner product (see [13]). Consequently, all eigenvalues of $K$ are real-valued. Even more can be stated about its spectrum: From

$$
\int_{\Omega} \bar{\psi}(-\mathrm{i} \boldsymbol{\nabla}-\boldsymbol{A})^{2} \varphi=\int_{\Omega} \overline{(-\mathrm{i} \boldsymbol{\nabla}-\boldsymbol{A}) \psi}(-\mathrm{i} \boldsymbol{\nabla}-\boldsymbol{A}) \varphi-\mathrm{i} \int_{\partial \Omega} \bar{\psi} \mathbf{n} \cdot(-\mathrm{i} \boldsymbol{\nabla}-\boldsymbol{A}) \varphi
$$

for all $\psi, \varphi \in L_{\mathbb{C}}^{2}(\Omega)$, it follows that the kinetic energy operator is positivesemidefinite over the subspace $\widetilde{X} \subseteq X$,

$$
\widetilde{X}:=\{\psi \in X: \mathbf{n} \cdot(-\mathrm{i} \boldsymbol{\nabla}-\boldsymbol{A}) \psi=0 \text { a.e. on } \partial \Omega\} .
$$

This is because for all $\psi \in \tilde{X}$,

$$
\langle\psi, K \psi\rangle_{L^{2}(\Omega)}=\int_{\Omega} \bar{\psi} K \psi \mathrm{d} \Omega=\int_{\Omega}\|(-\mathrm{i} \boldsymbol{\nabla}-\boldsymbol{A}) \psi\|^{2} \mathrm{~d} \Omega \geq 0 .
$$

Moreover, the value of 0 is attained if and only if

$$
(-\mathrm{i} \boldsymbol{\nabla}-\boldsymbol{A}) \psi=\mathbf{0} \quad \text { a.e. on } \Omega,
$$


from which in turn follows that

$$
\begin{array}{r}
\mathbf{0}=\boldsymbol{\nabla} \times(-\mathrm{i} \boldsymbol{\nabla} \psi)-\boldsymbol{\nabla} \times(\boldsymbol{A} \psi) \\
=-\mathrm{i}(\boldsymbol{\nabla} \times \boldsymbol{\nabla} \psi)-(\boldsymbol{\nabla} \times \boldsymbol{A}) \psi-(\boldsymbol{\nabla} \psi) \times \boldsymbol{A} \\
=-\boldsymbol{B} \psi-\mathrm{i}(\boldsymbol{A} \psi) \times \boldsymbol{A}=-\boldsymbol{B} \psi \quad \text { a.e. on } \Omega .
\end{array}
$$

Hence, only for vanishing magnetic fields $\boldsymbol{B}$, the kinetic energy operator $K$ is actually degenerate.

An approximation for the smallest magnitude eigenvalue around the constant zero-field $\boldsymbol{A}_{0} \equiv \mathbf{0}$ can be obtained by eigenvalue perturbation. Note that $K\left(\boldsymbol{A}_{0}\right)$ is the Laplace operator with homogeneous Neumann boundary conditions, so the smallest magnitude eigenvalue of $K\left(\boldsymbol{A}_{0}\right)$ is $\lambda_{0}=0$, the corresponding constant eigenfunction $v_{0} \equiv 1$. For the perturbed problem $\left(K\left(\boldsymbol{A}_{0}\right)+\delta K\right)\left(v_{0}+\delta v\right)=$ $\left(\lambda_{0}+\delta \lambda\right)\left(v_{0}+\delta v\right)$, one gets

$$
\delta \lambda\left\langle v_{0}, v_{0}\right\rangle=\left\langle v_{0},(\delta K) v_{0}\right\rangle+\left\langle v_{0},(\delta K-I \delta \lambda) \delta v\right\rangle,
$$

such that, in first-order approximation,

$$
\delta \lambda \approx \frac{\left\langle v_{0},(\delta K) v_{0}\right\rangle}{\left\langle v_{0}, v_{0}\right\rangle}=\frac{\left\langle v_{0},\left(K(\boldsymbol{A})-K\left(\boldsymbol{A}_{0}\right)\right) v_{0}\right\rangle}{\left\langle v_{0}, v_{0}\right\rangle}
$$

Noting that $K(\boldsymbol{A}) v_{0}=(-\mathrm{i} \boldsymbol{\nabla}-\boldsymbol{A})^{2} v_{0}=\boldsymbol{A}^{2} v_{0}$, this yields

$$
\lambda \approx \lambda_{0}+\frac{\left\langle v_{0}, \boldsymbol{A}^{2} v_{0}\right\rangle}{\left\langle v_{0}, v_{0}\right\rangle}=|\Omega|^{-1} \int_{\Omega} \boldsymbol{A}^{2} .
$$

This shows a lot more of the structure of the Jacobian operator $J(\psi)$ already: For any given $\psi \in X, J(\psi)$ is the composition of a self-adjoint, positive(semi)definite operator and some reaction terms.

It is possible to infer certain properties of $J$ starting from here. From a numerical point of view, insight into the adjointness and the spectrum of the operator will be highly desirable. The peculiar structure of $J(\psi)$, acting on $\varphi$ and its pointwise complex conjugate, together with the inner product $(3)$ in $X$, yield

Lemma 1. For any given $\psi \in L^{2}(\Omega)$, the Jacobian operator $J(\psi)(5)$ is linear and self-adjoint with respect to the inner product (3).

ProOF. See 13 .

Now that the spectrum of $J(\psi)$ is known to be a subset of $\mathbb{R}$ as well, the natural question to ask is whether or not $J(\psi)$ is generally definite. Unfortunately, no such thing is true. Quite the contrary: Note that, for any solution $\psi_{\mathrm{s}}$ of (2), we have

$$
\begin{aligned}
J\left(\psi_{\mathrm{s}}\right)\left(\mathrm{i} \psi_{\mathrm{s}}\right)=\left[(-\mathrm{i} \boldsymbol{\nabla}-\boldsymbol{A})^{2}-1+2\left|\psi_{\mathrm{s}}\right|^{2}\right]\left(\mathrm{i} \psi_{\mathrm{s}}\right)-\mathrm{i} \psi_{\mathrm{s}}^{2} \overline{\psi_{\mathrm{s}}} \\
=\left(1-\left|\psi_{\mathrm{s}}\right|^{2}\right)\left(\mathrm{i} \psi_{\mathrm{s}}\right)-\mathrm{i} \psi_{\mathrm{s}}+2 \overline{\mathrm{i}} \overline{\psi_{\mathrm{s}}} \psi_{\mathrm{s}}^{2}-\mathrm{i} \psi_{\mathrm{s}}^{2} \overline{\psi_{\mathrm{s}}}=0,
\end{aligned}
$$

and hence $\operatorname{span}\left\{\mathrm{i} \psi_{\mathrm{s}}\right\} \subseteq \operatorname{ker} J\left(\psi_{\mathrm{s}}\right)$. This is a direct consequence of the fact that $\mathcal{G L}(\psi) \sqrt{2}$ is invariant under the transformation $\widetilde{\psi}=\exp (\mathrm{i} \chi) \psi$ for any $\chi \in \mathbb{R}$.

Besides the fact that there is always a degenerate eigenvalue and that all eigenvalues are real, not much more can be said about the spectrum; in general, 
$\mathcal{J}(\psi)$ is indefinite. The definiteness depends entirely on the state $\psi$; if $\psi$ is a solution to (2), it is said to be physically stable or unstable depending on whether or not $\mathcal{J}(\psi)$ has positive eigenvalues. Typically, solutions with relatively low energy tend to be stable whereas solutions with relatively high energy tend to be unstable.

\section{Discretization in finite volumes and link variables}

In recent years, the research in applications for superconductors has taken strong interest in studying the effect of the sample geometry on superconductivity phenomena, for example, of dents or holes in a domain. Such geometries cannot be captured well by classical Cartesian staggered grids [13], so finite element and finite volume approaches have been developed that incorporate properties of the continuous Ginzburg-Landau equations such as the gauge invariance. In [17, the method has been described for two-dimensional domains and shall be described here in general terms.

Let $\boldsymbol{x}_{j} \in \mathbb{R}^{d}, d \in\{2,3\}, j \in\{1, \ldots, n\}$ be a given set of discretization points at which states $\psi \in X$ will be approximated by $\psi_{j}^{(h)} \approx \psi\left(\boldsymbol{x}_{j}\right), \psi^{(h)} \in \mathbb{C}^{n}$. Each discretization point $\boldsymbol{x}_{j}$ be equipped with its corresponding Voronoi region,

$$
V_{j}:=\left\{\boldsymbol{x} \in \mathbb{R}^{d}:\left\|\boldsymbol{x}-\boldsymbol{x}_{j}\right\|<\left\|\boldsymbol{x}-\boldsymbol{x}_{k}\right\| \forall k \neq j\right\} .
$$

The set $\left\{V_{j}\right\}_{j=1}^{n}$ is referred to as Voronoi tessellation corresponding to the generator set $\left\{\boldsymbol{x}_{j}\right\}_{j=1}^{n}$. The dual to a Voronoi tessellation consists of simplices and is referred to as Delaunay triangulation $\left\{T_{i}\right\}_{i=1}^{m}$ (see, e.g., figure 3).

For the domain $\Omega^{(h)}:=\bigcup_{j=1}^{n} V_{j}=\bigcup_{i=1}^{m} T_{i}$, the significant part of the Gibbs energy (1) can be written as

$$
F(\psi, \boldsymbol{A})=\underbrace{\sum_{i=1}^{m} \int_{T_{i}}\|-\mathrm{i} \boldsymbol{\nabla} \psi-\boldsymbol{A} \psi\|^{2}}_{=: F_{1}(\psi, \boldsymbol{A})}+\underbrace{\sum_{j=1}^{n} \int_{V_{j}}\left(-|\psi|^{2}+\frac{1}{2}|\psi|^{4}\right)}_{=: F_{2}(\psi)} .
$$

The second term, $F_{2}$, is readily discretized by mass lumping,

$$
F_{2}^{(h)}\left(\psi^{(h)}\right):=\sum_{j=1}^{n}\left|V_{j}\right|\left(-\left|\psi_{j}^{(h)}\right|^{2}+\frac{1}{2}\left|\psi_{j}^{(h)}\right|^{4}\right) .
$$

For the discretization of $F_{1}$, we will first refer to a technique for triangular meshes in [17, extended to arbitrary dimension here.

Lemma 2. Let $\boldsymbol{e}_{i}, i \in\{1, \ldots, n\}$, with $n:=d(d+1) / 2$ be the edges of a nondegenerate $d$-dimensional simplex. Then the symmetric rank-1 matrices $\left\{\boldsymbol{e}_{i} \boldsymbol{e}_{i}^{\mathrm{T}}\right\}_{i=1}^{n}$ form a basis of the vector space of symmetric $d \times d$-matrices.

Proof. The number $n$ of edges in a $d$-dimensional simplex coincides with the dimensionality of the vector space of symmetric $d \times d$-matrices. Hence, only linear independence has to be shown. Assume then that

$$
0_{d, d}=\sum_{i=1}^{n} \beta_{i}\left(\boldsymbol{e}_{i} \boldsymbol{e}_{i}^{\mathrm{T}}\right)
$$


with some $\beta \in \mathbb{R}^{n}$. Since the simplex is not degenerate, there is a regular matrix $U$ that maps the edges $\left\{\boldsymbol{e}_{i}\right\}_{i=1}^{n}$ onto the edges $\left\{\tilde{\boldsymbol{e}}_{i}\right\}_{i=1}^{n}$ of the unit simplex. With this, (11) is equivalent to

$$
0_{d, d}=U\left(\sum_{i=1}^{n} \beta_{i}\left(\boldsymbol{e}_{i} \boldsymbol{e}_{i}^{\mathrm{T}}\right)\right) U^{\mathrm{T}}=\sum_{i=1}^{n} \beta_{i}\left(U \boldsymbol{e}_{i}\left(U \boldsymbol{e}_{i}\right)^{\mathrm{T}}\right)=\sum_{i=1}^{n} \beta_{i}\left(\tilde{\boldsymbol{e}}_{i} \tilde{\boldsymbol{e}}_{i}^{\mathrm{T}}\right) .
$$

For the edges parallel to one of the axes, we have $\tilde{\boldsymbol{e}}_{i} \tilde{\boldsymbol{e}}_{i}^{\mathrm{T}}=e_{k} e_{k}^{\mathrm{T}}$ with $e_{k}$ being the unit vector in $k$-direction. For the edges between the two axes $k_{1}, k_{2}$, we have $\tilde{\boldsymbol{e}}_{i} \tilde{\boldsymbol{e}}_{i}^{\mathrm{T}}=\left(e_{k_{1}}-e_{k_{2}}\right)\left(e_{k_{1}}-e_{k_{2}}\right)^{\mathrm{T}}$. As the matrix that belongs to edge between $k_{1}$, $k_{2}$ is the only matrix with a nonzero entry at $\left(k_{1}, k_{2}\right)$ (namely -1 ), its coefficient in 12 must be 0 . Similarly, the same holds for all other coefficients, such that (11) can only be fulfilled of $\beta_{i}=0$ for all $i \in\{1, \ldots, n\}$. Hence, the matrices $\left\{\boldsymbol{e}_{i} \boldsymbol{e}_{i}^{\mathrm{T}}\right\}_{i=1}^{n}$ are linearly independent.

Since the $\left\{\boldsymbol{e}_{i} \boldsymbol{e}_{i}^{\mathrm{T}}\right\}_{i=1}^{n}$ form a basis, there exists in particular a unique set of coefficients $a \in \mathbb{R}^{n}$, such that

$$
I_{d, d}=\sum_{\text {edges } \boldsymbol{e}_{i}} a_{i}\left(\boldsymbol{e}_{i} \boldsymbol{e}_{i}^{\mathrm{T}}\right) .
$$

From this, we immediately conclude

Corollary 3. Given a nondegenerate simplex $S \in \mathbb{R}^{d}$ with edges $\boldsymbol{e}_{i, j}:=\boldsymbol{x}_{i}-\boldsymbol{x}_{j}$, $i, j \in\{1, \ldots, d+1\}, i \neq j$, there are coefficients $\alpha_{i, j}$ such that

$$
\int_{S}\|\boldsymbol{u}\|_{2}^{2}=|S|\langle\boldsymbol{u}, \boldsymbol{u}\rangle_{2}=\sum_{\text {edges } \boldsymbol{e}_{i, j}} \alpha_{i, j}\left\langle\boldsymbol{u}, \boldsymbol{e}_{i, j}\right\rangle_{2}\left\langle\boldsymbol{e}_{i, j}, \boldsymbol{u}\right\rangle_{2}=\sum_{\text {edges } \boldsymbol{e}_{i, j}} \alpha_{i, j}\left|\left\langle\boldsymbol{e}_{i, j}, \boldsymbol{u}\right\rangle_{2}\right|^{2}
$$

for any $\boldsymbol{u} \in \mathbb{C}^{d}$.

Given a simplex, one way of determining the edge coefficients $\alpha_{i, j}$ is to solve the symmetric and positive-definite linear equation system $M \alpha=b$ with

$$
M_{i, j}:=\left\langle\boldsymbol{e}_{i}, \boldsymbol{e}_{j}\right\rangle_{2}^{2}, \quad b_{i}:=|S|\left\|\boldsymbol{e}_{i}\right\|_{2}^{2},
$$

where the edges are indexed subsequently.

Remark 1. For triangles, the edge coefficients $\alpha_{i, j}$ are explicitly given by

$$
\alpha_{i, j}=\frac{1}{2} \cot \theta_{i, j}=\frac{1}{2} \frac{t_{i, j}}{\sqrt{1-t_{i, j}^{2}}},
$$

where $\theta_{i, j}$ is the angle opposing the edge $\boldsymbol{e}_{i, j}\left[17\right.$, and $t_{i, j}:=\left\langle\frac{\boldsymbol{e}_{i, k}}{\left\|\boldsymbol{e}_{i, k}\right\|}, \frac{\boldsymbol{e}_{j, k}}{\left\|\boldsymbol{e}_{j, k}\right\|}\right\rangle_{2}$ with $k \notin\{i, j\}$. by

With corollary 3 (and the coefficients $\alpha_{i, j}$ from there), $F_{1}$ can be approximated

$$
F_{1}(\psi, \boldsymbol{A}) \approx \widehat{F}_{1}(\psi, \boldsymbol{A}):=\sum_{i=1}^{m} \sum_{\text {edges }} \alpha_{\boldsymbol{e}_{j, k} \text { of } T_{i}}^{(i)}\left|\boldsymbol{e}_{j, k} \cdot(-\mathrm{i} \boldsymbol{\nabla}-\boldsymbol{A}) \psi\left(\overline{\boldsymbol{x}}_{j, k}\right)\right|^{2}
$$


with $\overline{\boldsymbol{x}}_{j, k}:=\frac{1}{2}\left(\boldsymbol{x}_{j}+\boldsymbol{x}_{k}\right)$, or, more compactly,

$$
\widehat{F}_{1}(\psi, \boldsymbol{A})=\sum_{\text {edges } \boldsymbol{e}_{j, k}} \alpha_{j, k}\left|\boldsymbol{e}_{j, k} \cdot(-\mathrm{i} \boldsymbol{\nabla}-\boldsymbol{A}) \psi\left(\overline{\boldsymbol{x}}_{j, k}\right)\right|^{2}
$$

with the edge coefficients

$$
\alpha_{j, k}:=\sum_{\begin{array}{c}
\text { simplices } T_{i} \\
\text { adjacent to edge }
\end{array}} \alpha_{j, k}^{(i)} .
$$

One could now do a finite difference approximation in the differential terms of 13 to receive a Gibbs energy defined over the discretized function space $\mathbb{C}^{n}$. Note, however, that such naive discretization schemes of the momentum operator $-\mathrm{i} \boldsymbol{\nabla}-\boldsymbol{A}$ lead to systems that preserve gauge invariance - inherent to the Ginzburg-Landau equations - only up to a certain order in the spatial discretization. It is hence customary to rewrite the momentum operator in terms of variables that ensure preservation of gauge invariance for any pointwise discretization. Following [16, for any given normalized spatial direction $\boldsymbol{v}$, let

$$
U_{\boldsymbol{v}}(\boldsymbol{x}):=\exp \left(-\mathrm{i} \int_{\hat{\boldsymbol{x}}}^{\boldsymbol{x}} \boldsymbol{v} \cdot \boldsymbol{A}(\boldsymbol{w}) \mathrm{d} \boldsymbol{w}\right)
$$

with arbitrary, fixed $\hat{\boldsymbol{x}} \in \boldsymbol{x}+\operatorname{span}\{\boldsymbol{v}\}$ (e.g., $\hat{\boldsymbol{x}}=\boldsymbol{x}-(\boldsymbol{x} \cdot \boldsymbol{v}) \boldsymbol{v})$. Since $U_{\boldsymbol{v}}(\boldsymbol{x})$ sits on the unit circle, one has $\overline{U_{\boldsymbol{v}}(\boldsymbol{x})} U_{\boldsymbol{v}}(\boldsymbol{x})=1$, and with this

$$
\bar{U}_{\boldsymbol{v}} \boldsymbol{v} \cdot \boldsymbol{\nabla}\left(U_{\boldsymbol{v}} \psi\right) \equiv \bar{U}_{\boldsymbol{v}}\left(-\mathrm{i} \boldsymbol{v} \cdot \boldsymbol{A} U_{\boldsymbol{v}} \psi+U_{\boldsymbol{v}} \boldsymbol{v} \cdot \boldsymbol{\nabla} \psi\right) \equiv \mathrm{i} \boldsymbol{v} \cdot(-\mathrm{i} \boldsymbol{\nabla}-\boldsymbol{A}) \psi
$$

Thus, $\widehat{F}_{1}$ can be written as

$$
\widehat{F}_{1}(\psi, \boldsymbol{A})=\sum_{\text {edges } \boldsymbol{e}_{j, k}} \alpha_{j, k}\left|\overline{U_{\boldsymbol{e}_{j, k}}}\left(\overline{\boldsymbol{x}}_{j, k}\right) \boldsymbol{e}_{j, k} \cdot \boldsymbol{\nabla}\left(U_{\boldsymbol{e}_{j, k}} \psi\right)\left(\overline{\boldsymbol{x}}_{j, k}\right)\right|^{2} .
$$

Finite difference approximation finally yields the discretization

$$
\begin{aligned}
F_{1}^{(h)}\left(\psi^{(h)}, \boldsymbol{A}\right) & :=\sum_{\text {edges } \boldsymbol{e}_{j, k}} \alpha_{j, k}\left|\overline{U_{\boldsymbol{e}_{j, k}}}\left(\overline{\boldsymbol{x}}_{j, k}\right)\left(U_{\boldsymbol{e}_{j, k}}\left(\boldsymbol{x}_{j}\right) \psi_{j}^{(h)}-U_{\boldsymbol{e}_{j, k}}\left(\boldsymbol{x}_{k}\right) \psi_{k}^{(h)}\right)\right|^{2} \\
& =\sum_{\text {edges } \boldsymbol{e}_{j, k}} \alpha_{j, k}\left|U_{j, k} \psi_{j}^{(h)}-\psi_{k}^{(h)}\right|^{2}
\end{aligned}
$$

with

$$
U_{j, k}:=\exp \left(-\mathrm{i} \int_{\boldsymbol{x}_{k}}^{\boldsymbol{x}_{j}} \boldsymbol{e}_{j, k} \cdot \boldsymbol{A}(\boldsymbol{w}) \mathrm{d} \boldsymbol{w}\right),
$$

often called link-variable [16. If $\boldsymbol{A}$ is known only at certain points of along the edges, $U_{j, k}$ could again be approximated by a quadrature formula.

Finally, together with 10$]$, the discrete Ginzburg-Landau energy functional is defined as

$$
F^{(h)}\left(\psi^{(h)}, \boldsymbol{A}\right):=F_{1}^{(h)}\left(\psi^{(h)}, \boldsymbol{A}\right)+F_{2}^{(h)}\left(\psi^{(h)}\right) .
$$


The standard Euler-Lagrange formalism now yields a necessary condition for extremal points of the energy functional,

$$
\begin{gathered}
0=2 \Re\left(\sum_{\text {edges } \boldsymbol{e}_{j, k}} \alpha_{j, k}\left[\left(\psi_{j}^{(h)}-U_{j, k} \psi_{k}^{(h)}\right) \overline{\delta \psi_{j}^{(h)}}+\left(\psi_{k}^{(h)}-\overline{U_{j, k}} \psi_{j}^{(h)}\right) \overline{\delta \psi_{k}^{(h)}}\right]\right. \\
\left.-\sum_{j=1}^{n}\left|V_{j}\right| \psi_{j}^{(h)}\left(1-\left|\psi_{j}^{(h)}\right|^{2}\right) \overline{\delta \psi_{j}^{(h)}}\right) \quad \forall \delta \psi^{(h)} \in \mathbb{C}^{n} .
\end{gathered}
$$

This is equivalent to the discrete Ginzburg-Landau equations,

$$
\forall i \in\{1, \ldots, n\}: \quad 0=\left(K^{(h)} \psi^{(h)}\right)_{i}-\psi_{i}^{(h)}\left(1-\left|\psi_{i}^{(h)}\right|^{2}\right),
$$

where the discrete kinetic energy operator $K^{(h)}$ is defined by

$$
\begin{aligned}
& \forall \phi^{(h)}, \psi^{(h)} \in \mathbb{C}^{n}: \quad\left\langle\phi^{(h)}, K^{(h)} \psi^{(h)}\right\rangle= \\
& \sum_{\text {edges } \boldsymbol{e}_{j, k}} \alpha_{j, k}\left[\left(\psi_{j}^{(h)}-U_{j, k} \psi_{k}^{(h)}\right) \bar{\phi}_{j}^{(h)}+\left(\psi_{k}^{(h)}-\overline{U_{j, k}} \psi_{j}^{(h)}\right) \bar{\phi}_{k}^{(h)}\right]
\end{aligned}
$$

with the discrete inner product

$$
\left\langle\psi^{(h)}, \phi^{(h)}\right\rangle:=\sum_{i=1}^{n}\left|V_{i}\right| \bar{\psi}_{i}^{(h)} \phi_{i}^{(h)} .
$$

Remark 2. In matrix form, the operator $K^{(h)}$ is represented as a product $K^{(h)}=D^{-1} \widehat{K}$ of the diagonal matrix $D^{-1}, D_{i, i}=\left|V_{i}\right|$, and a Hermitian matrix $\widehat{K}$.

The discretization (17) has several advantages, starting with the fact that the boundary conditions of the Ginzburg-Landau equations (2) are naturally contained. Also note that the discrete kinetic energy operator (18) coincides, up to the terms $U_{j, k}$, with the discretization of the Laplace operator with homogeneous Neumann boundary conditions. Similarly, it has a number of desirable properties that will make the iterative solution of the Jacobian system easier.

Lemma 4. The discrete kinetic energy operator $K^{(h)}$ (18) is self-adjoint with respect to the discrete inner product (19).

Proof. Let $\phi^{(h)}, \psi^{(h)} \in \mathbb{C}^{n}$. Then

$$
\begin{aligned}
\left\langle\phi^{(h)}, K^{(h)} \psi^{(h)}\right\rangle & =\sum_{\text {edges } \boldsymbol{e}_{j, k}} \alpha_{j, k}\left[\left(\psi_{j}^{(h)}-U_{j, k} \psi_{k}^{(h)}\right) \bar{\phi}_{j}^{(h)}+\left(\psi_{k}^{(h)}-\overline{U_{j, k}} \psi_{j}^{(h)}\right) \bar{\phi}_{k}^{(h)}\right] \\
& =\sum_{\text {edges } \boldsymbol{e}_{j, k}} \alpha_{j, k}\left[\overline{\left(\phi_{j}^{(h)}-U_{j, k} \phi_{k}^{(h)}\right)} \psi_{j}^{(h)}+\overline{\left(\phi_{k}^{(h)}-\overline{U_{j, k}} \phi_{j}^{(h)}\right)} \psi_{k}^{(h)}\right] \\
& =\left\langle K^{(h)} \phi^{(h)}, \psi^{(h)}\right\rangle .
\end{aligned}
$$

Lemma 5. The discrete kinetic energy operator $K^{(h)}(18)$ is positive-semidefinite. 
Proof. Let $\psi^{(h)} \in \mathbb{C}^{n}$. Then

$$
\begin{aligned}
\left\langle\psi^{(h)}, K^{(h)} \psi^{(h)}\right\rangle & =\sum_{\text {edges } \boldsymbol{e}_{j, k}} \alpha_{j, k}\left[\left(\psi_{j}^{(h)}-U_{j, k} \psi_{k}^{(h)}\right) \bar{\psi}_{j}^{(h)}+\left(\psi_{k}^{(h)}-\overline{U_{j, k}} \psi_{j}^{(h)}\right) \bar{\psi}_{k}^{(h)}\right] \\
& =\sum_{\text {edges } \boldsymbol{e}_{j, k}} \alpha_{j, k}\left[\psi_{j}^{(h)} \bar{\psi}_{j}^{(h)}-U_{j, k} \psi_{k}^{(h)} \bar{\psi}_{j}^{(h)}+\psi_{k}^{(h)} \bar{\psi}_{k}^{(h)}-\bar{U}_{j, k} \psi_{j}^{(h)} \bar{\psi}_{k}^{(h)}\right] .
\end{aligned}
$$

Noting that $U_{j, k} \overline{U_{j, k}}=1$, this yields

$$
\left\langle\psi^{(h)}, K^{(h)} \psi^{(h)}\right\rangle=\sum_{\text {edges } \boldsymbol{e}_{j, k}} \alpha_{j, k}\left|\psi_{j}^{(h)}-U_{j, k} \psi_{k}^{(h)}\right|^{2} \geq 0
$$

For $\boldsymbol{A}_{0} \equiv \mathbf{0}$, the state $\widehat{\psi}^{(h)} \equiv 1$ is obviously an eigenvector of $K^{(h)}$ with the eigenvalue 0. Equation (20) also delivers an approximation $\tilde{\lambda}_{0}$ for the smallest-magnitude eigenvalue for perturbations of $\boldsymbol{A}_{0}$, namely

$$
\begin{aligned}
\tilde{\lambda}_{0}=\frac{\left\langle\widehat{\psi}^{(h)}, K^{(h)} \widehat{\psi}^{(h)}\right\rangle}{\left\langle\widehat{\psi}^{(h)}, \widehat{\psi}^{(h)}\right\rangle} & =\left|\Omega^{(h)}\right|^{-1} \sum_{\text {edges } \boldsymbol{e}_{j, k}} \alpha_{j, k}\left|1-U_{j, k}\right|^{2} \\
& =\left|\Omega^{(h)}\right|^{-1} \sum_{\text {edges } \boldsymbol{e}_{j, k}} \alpha_{j, k}\left|2 \sin \left(\frac{\arg U_{j, k}}{2}\right)\right|^{2},
\end{aligned}
$$

or, in first approximation,

$$
\begin{aligned}
\tilde{\lambda}_{0} & \approx\left|\Omega^{(h)}\right|^{-1} \sum_{\text {edges } \boldsymbol{e}_{j, k}} \alpha_{j, k}\left|\arg U_{j, k}\right|^{2} \\
& =\left|\Omega^{(h)}\right|^{-1} \sum_{\text {edges } \boldsymbol{e}_{j, k}} \alpha_{j, k}\left|\int_{\boldsymbol{x}_{k}}^{\boldsymbol{x}_{j}} \boldsymbol{e}_{j, k} \cdot \boldsymbol{A}(\boldsymbol{w}) \mathrm{d} \boldsymbol{w}\right|^{2}
\end{aligned}
$$

Compare this with the corresponding continuous expression (8).

Completely analogous to the results for the continuous Jacobian operator $J(\psi)$, the discrete Jacobian operator

$$
\left(J^{(h)}\left(\psi^{(h)}\right) \phi^{(h)}\right)_{i}:=\left(K^{(h)} \phi^{(h)}\right)_{i}+\left(-1+2\left|\psi_{i}^{(h)}\right|^{2}\right) \phi_{i}^{(h)}+\left(\psi_{i}^{(h)}\right)^{2} \bar{\phi}_{i}^{(h)}
$$

of 17] is self-adjoint with respect to the inner product

$$
\left\langle\phi^{(h)}, \psi^{(h)}\right\rangle_{\mathbb{R}}:=\Re\left\langle\phi^{(h)}, \psi^{(h)}\right\rangle .
$$

Like the continuous Jacobian operator $J(\psi), J^{(h)}\left(\psi^{(h)}\right)$ also has a nontrivial kernel if $\psi^{(h)}$ is a solution to the problem. While in the Newton process, the Jacobian system will never need to be solved in exactly a solution, states close to a solution might introduce numerical difficulties when nearly-singular systems need to be solved. Techniques for this situation include adding phase conditions [13] or deflation methods. 
Remark 3. Note that there is a vector space isometry of $\mathbb{C}^{n}$ as vector space over the field $\mathbb{R}$ and $\mathbb{R}^{2 n}$ with its natural inner product: For all $\phi^{(h)}, \psi^{(h)} \in \mathbb{C}^{n}$,

$$
\left\langle\left(\begin{array}{c}
\Re \phi^{(h)} \\
\Im \phi^{(h)}
\end{array}\right),\left(\begin{array}{l}
\Re \psi^{(h)} \\
\Im \psi^{(h)}
\end{array}\right)\right\rangle=\left\langle\Re \phi^{(h)}, \Re \psi^{(h)}\right\rangle+\left\langle\Im \phi^{(h)}, \Im \psi^{(h)}\right\rangle=\left\langle\phi^{(h)}, \psi^{(h)}\right\rangle_{\mathbb{R}} .
$$

This is relevant in practice if the complex-valued original problem $(17)$ in $\mathbb{C}^{n}$ is implemented in terms of $\mathbb{R}^{2 n}$. Using the natural inner product in this space will yield the expected results without having to take particular care of $\langle\cdot, \cdot\rangle_{\mathbb{R}}$.

\section{Algorithm and numerical results}

For real-world three-dimensional domains, the solution of the discrete equivalent of the Jacobian system (4) will have too many unknowns for black-box strategies such as $L U$-decompositions to fit into memory. Exploiting the sparsity structure of the operator is crucial, and hence Krylov subspace methods are an attractive alternative. The choice of the appropriate Krylov subspace method depends on the spectral properties of the operator. Its self-adjointness (see lemma 1 and its discrete equivalent) make it possible for symmetric Krylov subspace methods to efficiently solve the linear system if adapted for the inner product 22 (see also remark 3). This avoids the larger memory-requirements that come with asymmetric solvers such as GMRES. Furthermore, as $J^{(h)}\left(\psi^{(h)}\right)$ is generally indefinite (depending on $\psi^{(h)}$ ) and the number of negative eigenvalues can be large, CG may be unsuitable as a solver. While it is known to perform well for indefinite problems when the number of negative eigenvalues is not too large [18, convergence can be irregular. In contrast, MINRES is designed to deal with indefinite systems and is hence a more suitable choice.

\subsection{Preconditioning}

As the main computational effort of the nonlinear solver flows into the linear solves of the Jacobian system, and the complexity of the linear solve usually grows faster than linearly with the number of unknowns in the system, it is crucial to explore the possibilities of accelerating the Krylov solver using an appropriate preconditioner. Given the results of section 2.1. we will evaluate the use of approximate inverses of the operator

$$
P^{(h)}\left(\psi^{(h)}\right):=K^{(h)}+2\left|\psi^{(h)}\right|^{2}
$$

as a preconditioner for $J^{(h)}\left(\psi^{(h)}\right)$. The operator $P^{(h)}\left(\psi^{(h)}\right)$ is obviously selfadjoint with respect to the standard discrete inner product $\sqrt{19}$ and positivesemidefinite. From lemma 5 , we can conclude that it is even strictly positivedefinite except for the uninteresting case $\psi^{(h)} \equiv 0, \boldsymbol{A} \equiv \mathbf{0}$. Moreover, $K^{(h)}$ is derived from a geometric discretization, and its sparsity structure coincides with that of the Laplacian with homogeneous Neumann boundary conditions. This makes the inversion of $P^{(h)}\left(\psi^{(h)}\right)$ a suitable target for algebraic-multigrid (AMG) strategies which are known to yield optimal convergence behavior in the sense that the number of iterations required to reach a certain stopping criterion is independent of the number of unknowns in the system. Furthermore, AMG methods are memory-efficient and scale well in parallel computing environments [19, 20, 21]. The only nonstandard circumstance here is the fact that the matrix entries are complex-valued. Difficulties in this area, however, were discussed and treated in 22 . 
Remark 4. The operator $Q^{(h)}\left(\psi^{(h)}\right)$ defined by

$$
\begin{aligned}
Q^{(h)}\left(\psi^{(h)}\right) \phi^{(h)} & :=\left(J^{(h)}\left(\psi^{(h)}\right)+I\right) \phi^{(h)} \\
& =\left(K^{(h)}+2\left|\psi^{(h)}\right|^{2}\right) \phi^{(h)}+\left(\psi^{(h)}\right)^{2} \overline{\phi^{(h)}} \quad \forall \phi^{(h)} \in \mathbb{C}^{n}
\end{aligned}
$$

is obviously self-adjoint and also positive-definite since

$$
\begin{aligned}
& \left\langle\phi^{(h)}, 2\left|\psi^{(h)}\right|^{2} \phi^{(h)}+\left(\psi^{(h)}\right)^{2} \bar{\phi}^{(h)}\right\rangle_{\mathbb{R}} \\
& =\Re\left[\sum_{i=1}^{n}\left|V_{i}\right|\left(\bar{\phi}_{i}^{(h)} \cdot 2\left|\psi_{i}^{(h)}\right|^{2} \phi_{i}^{(h)}+\bar{\phi}_{i}^{(h)} \cdot\left(\psi_{i}^{(h)}\right)^{2} \bar{\phi}_{i}^{(h)}\right)\right] \\
& =\sum_{i=1}^{n}\left|V_{i}\right|\left(2\left|\psi_{i}^{(h)} \bar{\phi}_{i}^{(h)}\right|^{2}+\Re\left(\psi_{i}^{(h)} \bar{\phi}_{i}^{(h)}\right)^{2}\right) \\
& >0 .
\end{aligned}
$$

It would hence also be a candidate for a good preconditioner. However, unlike $P^{(h)}$, it cannot be represented as a matrix and is thus not suitable for solution with AMG.

Note that the operator $\mathrm{AMG}_{k}(A, b)$, defined by $k$ AMG cycles applied to a Hermitian problem $A x=b$, is again Hermitian. With remark 2 (and $D$ from there), we have that

$$
\left(P^{(h)}\left(\psi^{(h)}\right)\right)^{-1}=\left(\widehat{K}+2 D\left|\psi^{(h)}\right|^{2}\right)^{-1} D
$$

so the approximate inverse of $P^{(h)}\left(\psi^{(h)}\right)$,

$$
R_{k}^{(h)}\left(\psi^{(h)}\right)=\operatorname{AMG}_{k}\left(\widehat{K}+2 D\left|\psi^{(h)}\right|^{2}, D \cdot\right),
$$

is self-adjoint with respect to the standard discrete inner product $(19)$.

We will now explore this idea through numerical experiments, for the preconditioners $R_{1}^{(h)}\left(\psi^{(h)}\right)$ and

$$
R_{\infty}^{(h)}\left(\psi^{(h)}\right):=\left(P^{(h)}\left(\psi^{(h)}\right)\right)^{-1}=\left(\widehat{K}+2 D\left|\psi^{(h)}\right|^{2}\right)^{-1} D,
$$

where $\widehat{K}+2 D\left|\psi^{(h)}\right|^{2}$ is inverted numerically with high accuracy.

It is notoriously difficult to rigorously characterize the spectrum of the Jacobian operator of the Ginzburg-Landau problem, and the situation is similar for the preconditioned operator. Nevertheless, if $\left(\lambda, \phi^{(h)}\right)$ is an eigenvalue/eigenvector pair of the preconditioned operator $R_{\infty}^{(h)}\left(\psi^{(h)}\right) J^{(h)}\left(\psi^{(h)}\right)$, i.e.,

$$
J^{(h)}\left(\psi^{(h)}\right) \phi^{(h)}=\lambda P^{(h)}\left(\psi^{(h)}\right) \phi^{(h)} .
$$

one gets

$$
\lambda=\frac{\left\langle\phi^{(h)}, J^{(h)}\left(\psi^{(h)}\right) \phi^{(h)}\right\rangle_{\mathbb{R}}}{\left\langle\phi^{(h)}, P^{(h)}\left(\psi^{(h)}\right) \phi^{(h)}\right\rangle_{\mathbb{R}}}=1+\frac{\Re\left\langle\left(\phi^{(h)}\right)^{2},\left(\psi^{(h)}\right)^{2}\right\rangle-1}{\left\langle\phi^{(h)}, K^{(h)} \phi^{(h)}\right\rangle+2\left\langle\left|\phi^{(h)}\right|^{2},\left|\psi^{(h)}\right|^{2}\right\rangle} .
$$



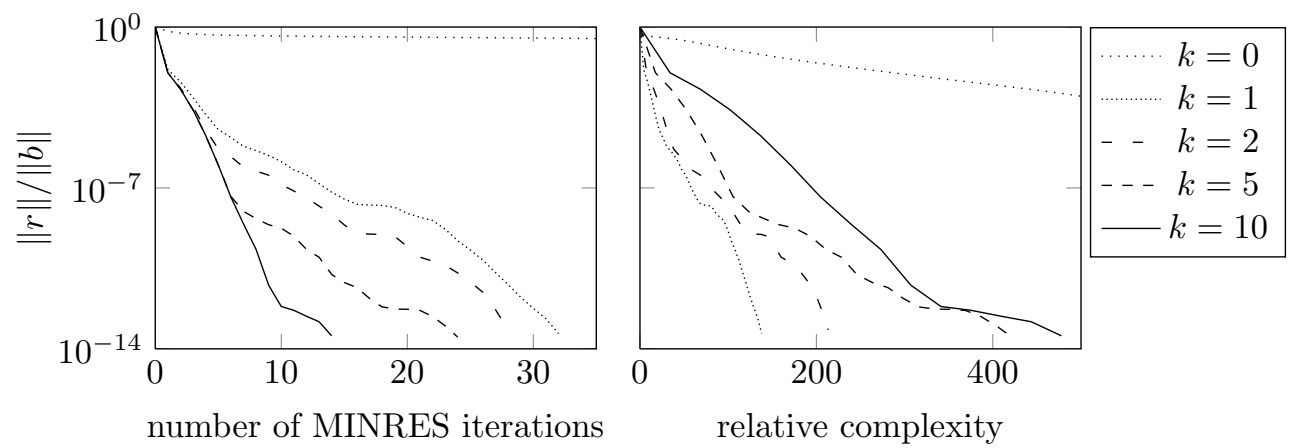

Figure 2: Typical residual behavior for MINRES, applied to the problem $J^{(h)}\left(\psi^{(h)}\right) \phi^{(h)}=b^{(h)}$, preconditioned with $R_{k}^{(h)}\left(\psi^{(h)}\right)$ for different $k$, with each $\psi^{(h)} \equiv b^{(h)} \equiv 1$, initial guess $\phi_{0}^{(h)} \equiv 0$, for $\Omega_{\mathrm{sq}}^{(h)}$ in $\boldsymbol{A}_{z}$. The number of unknowns is $1000^{2}$ in all cases. $10 \mathrm{AMG}$ steps solve the preconditioning problem in each MINRES step up to at least $\left\|r_{\mathrm{p}}\right\| \leq 10^{-12}$. In the figure on the right, the computational complexity is measured in terms of the cost of one matrix-vector multiplication. For this setting, the application of one V-cycle costs as much as about 3.31 matrix-vector multiplications.

In case $\left|\psi^{(h)}\right| \gg 1$ (which can happen during the Newton iteration), the eigenvalues cluster around $1 \pm \frac{1}{2}$ (depending on the sign of $\Re\left\langle\left(\phi^{(h)}\right)^{2},\left(\psi^{(h)}\right)^{2}\right\rangle$ ), so the preconditioned problem can be expected to be solved in a small number of Krylov iterations. Noting that solutions $\psi$ of the Ginzburg-Landau equations (2) fulfill $|\psi|<1$ pointwise, though, (25) unfortunately gives little insight in the behavior close to a solution. The same is true for the bounds gained from estimating the denominator term $\left\langle\phi^{(h)}, K^{(h)} \phi^{(h)}\right\rangle$ with the help of the smallest eigenvalue approximation for weak fields $(21)$.

While $R_{\infty}^{(h)}\left(\psi^{(h)}\right)$ is obviously more expensive to apply, it is expected that it will yield a smaller number of Krylov iterations as compared to preconditioning with $R_{1}^{(h)}\left(\psi^{(h)}\right)$. Figure 2 illustrates this: For a fixed setup, the preconditioners $R_{k}^{(h)}\left(\psi^{(h)}\right)$ with $k \in\{1,2,5,10\}$ are compared, where for this particular case $R_{10}^{(h)}\left(\psi^{(h)}\right) \approx R_{\infty}^{(h)}\left(\psi^{(h)}\right)$ to machine-precision. Preconditioning with $R_{10}^{(h)}\left(\psi^{(h)}\right)$ indeed results in the smallest number of required MINRES iterations; if fewer V-cycles are applied per iteration, the number of iterations increases. A better measure for the overall computational cost than the sheer number of Krylov iterations, however, is the number of performed V-cycles together with the matrix-vector products. While the latter mainly depends on the number of nonzeros in the kinetic energy operator $K^{(h)}$, the cost of the former also depends the many parameters of AMG. In all of the experiments performed in this paper, the cost of the application of one V-cycle is between three and four times the cost of a matrix-vector product of the corresponding matrix. As can be seen in the right panel of figure 2, no more than the equivalent of about 140 matrix-vector products is are required in total to converge the MINRES process in combination a single V-cycle preconditioning. At the same time, 10 cycles per step require the equivalent of about 480 matrix-vector multiplications. This points to the fact that the approximate inversion with a single V-cycle will lead to the fastest 
solver.

For the experiments in figure 2 and all experiments in the remainder of this paper, smoothed-aggregation AMG with one pre- and one post-smoothing step of symmetric Gauss-Seidel was used. The method is implemented using PyAMG 23.

We now look at the application to the two-dimensional regular polygons in the $x$-y-plane

$$
\begin{aligned}
& \Omega_{\mathrm{tri}}:=H_{\text {convex }}\left(\left\{\left(\begin{array}{l}
0 \\
5
\end{array}\right),\left(\begin{array}{c}
-5 \sqrt{3} / 2 \\
-5 / 2
\end{array}\right),\left(\begin{array}{c}
5 \sqrt{3} / 2 \\
-5 / 2
\end{array}\right)\right\}\right) \text { (figure 3a), } \\
& \Omega_{\mathrm{sq}}:=\left\{\boldsymbol{x}:\|\boldsymbol{x}\|_{\infty}<5 / \sqrt{2}\right\} \text { (figure 3b, } \\
& \Omega_{\text {circ }}:=\left\{\boldsymbol{x}:\|\boldsymbol{x}\|_{2}<5\right\} \text { (figure 3c), }
\end{aligned}
$$

and the three-dimensional regular polyhedra

$\Omega_{\text {tet }}:=5 \cdot H_{\text {convex }}\left(\left\{\left(\begin{array}{l}0 \\ 0 \\ 1\end{array}\right),\left(\begin{array}{c}2 \sqrt{2} / 3 \\ 0 \\ -1 / 3\end{array}\right),\left(\begin{array}{c}-\sqrt{2} / 3 \\ \sqrt{2 / 3} \\ -1 / 3\end{array}\right),\left(\begin{array}{c}-\sqrt{2} / 3 \\ -\sqrt{2 / 3} \\ -1 / 3\end{array}\right)\right\}\right)$ (figure 3d),

$\Omega_{\text {cube }}:=\left\{\boldsymbol{x}:\|\boldsymbol{x}\|_{\infty}<5 / \sqrt{3}\right\}$ (figure 3e),

$\Omega_{\text {ball }}:=\left\{\boldsymbol{x}:\|\boldsymbol{x}\|_{2}<5\right\}$ (figure 3f),

all centered at the origin with circumradius 5. For each domain, both the potentials

$$
\boldsymbol{A}_{z}(\boldsymbol{x}):=\frac{1}{2}(-y, x, 0)^{\mathrm{T}},
$$

representing the homogeneous field $\boldsymbol{B}=(0,0,1)^{\mathrm{T}}$, and

$$
\boldsymbol{A}_{\mathrm{d}}(\boldsymbol{x}):=\frac{1}{\left\|\boldsymbol{x}-\boldsymbol{x}_{0}\right\|^{3}}\left(\boldsymbol{m} \times\left(\boldsymbol{x}-\boldsymbol{x}_{0}\right)\right),
$$

representing the inhomogeneous field generated by a magnetic dipole at the location

$$
\boldsymbol{x}_{0}=\left\{\begin{array}{l}
(0,0,1)^{\mathrm{T}} \text { for the } 2 \mathrm{D} \text { domains } \\
(0,0,6)^{\mathrm{T}} \text { for the 3D domains }
\end{array}\right.
$$

and with the dipole moment $\boldsymbol{m}=(0,0,1)^{\mathrm{T}}$, are considered. For all experiments, we considered $\left.J^{(h)}\left(\psi^{(h)}\right)\right)$ with $\psi^{(h)} \equiv 1$. For other choices of $\psi^{(h)}$ see the paragraph on numerical continuation below.

Figures 4 and 5 show the number of MINRES iterations as a function of the dimension of the solution space. For the unpreconditioned system, the number of iteration increases as expected since the finer discretization makes the condition number of $K^{(h)}$ and hence $J^{(h)}$ larger. In contrast to this, when $R_{1}^{(h)}$ and $R_{\infty}^{(h)}$ are applied as preconditioners, the number of iterations remains bounded in all problem settings as the discretization refines. Although the number of iterations, when preconditioned with $R_{1}^{(h)}$, is slightly larger compared to preconditioning with $R_{\infty}^{(h)}$, the former is actually computationally cheaper as discussed above (see figure 2). These numerical experiments suggest that for various fixed domains and magnetic vector potentials, the number of iterations of the Krylov solver is independent of the number of unknowns. 


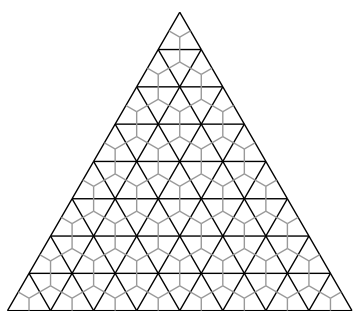

(a) $\Omega_{\text {tri }}^{(h)}$.

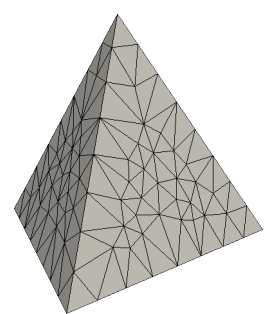

(d) $\Omega_{\text {tet }}^{(h)}$.

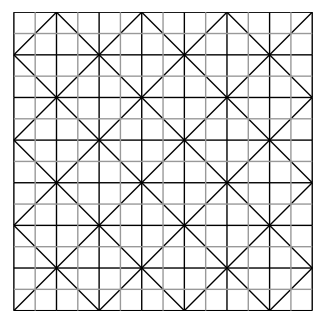

(b) $\Omega_{\mathrm{sq}}^{(h)}$.

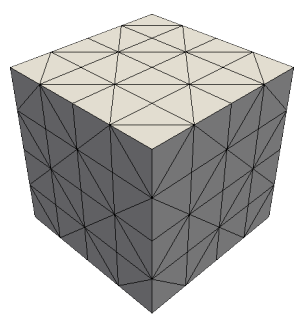

(e) $\Omega_{\text {cube }}^{(h)}$.

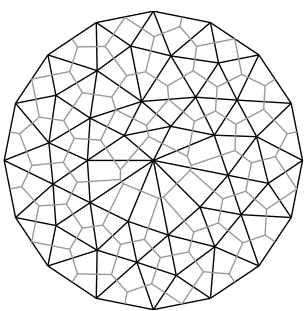

(c) $\Omega_{\text {circ }}^{(h)}$

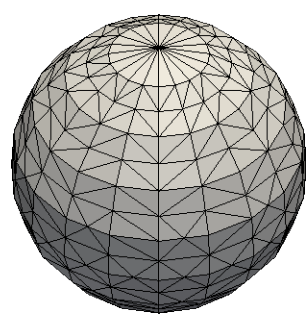

(f) $\Omega_{\mathrm{ball}}^{(h)}$

Figure 3: Triangulations of test domains, shown in rather coarse discretizations. For the two-dimensional domains, the Voronoi regions $V_{i}$ as appearing in (10) are highlighted in light gray.

Numerical parameter continuation. A common application of the Newton-Krylov solver is in a numerical continuation context where a family (or curve) of solution states (from a given state space $X$ ) is constructed as a function of a parameter (from a given parameter space $A$ ) in the system. This is a popular way of exploring the solution landscape of nonlinear equations, and amongst the most widely used algorithms for this purpose is pseudo-arclength continuation 12, a predictor-corrector method. Here, in each continuation step an initial guess is constructed as an extrapolation to the solution curve in $X \times A$ which is then corrected perpendicularly to the extrapolation, typically involving a NewtonKrylov process. In applications, many curves, each with thousands of solutions, are computed. Each continuation step requires the solution of a nonlinear system, each of which requires the solution of a Jacobian system.

As this setting presents a typical use case for the preconditioner introduced above, a representative problem is discussed in this section. As opposed to all previous numerical experiments, the state $\psi^{(h)}$ deviates significantly from the initial state $\psi_{0}^{(h)} \equiv 1$ in the corresponding numerical experiment (see figures $6 \mathrm{c}$ $6 \mathrm{~d}$.

Figure 6 illustrates the performance of the $R_{1}^{(h)}$-preconditioned Krylov-solver for $\Omega_{\mathrm{sq}}^{(h)}$ with edge length 10 in $\mu \boldsymbol{A}_{z} \sqrt{26}, \mu \in \mathbb{R}$. The strength $\mu$ of the magnetic field is used as continuation parameter, and the continuation is started with the trivial solution $\psi_{0}^{(h)} \equiv 1$ at $\mu=0$. As $\mu$ increases, the solution starts to deviate from the homogeneous state. Throughout the parameter continuation, vortices appear in the domain and the state loses its stability [13, a process marked by eigenvalues of the Jacobian crossing the origin, i.e., a change of definiteness of the Jacobian operator. The right panel of figure 6 shows, for each point on 


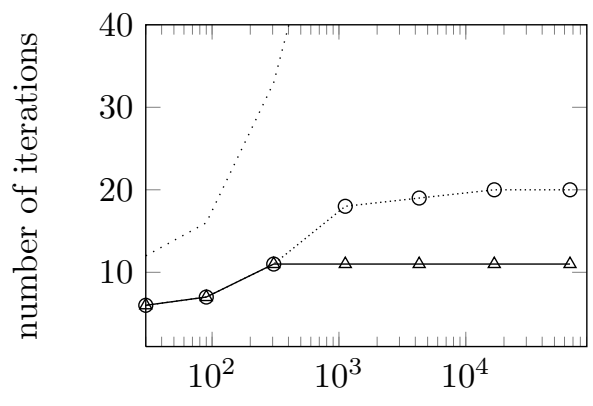

dimension of solution space

(a) Triangle in $\boldsymbol{A}_{z}$.

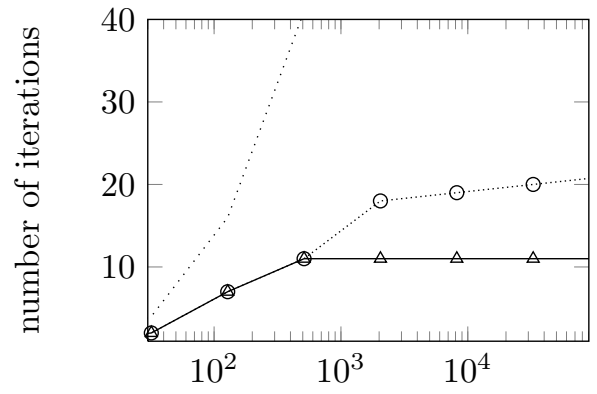

dimension of solution space

(c) Rectangle in $\boldsymbol{A}_{z}$.

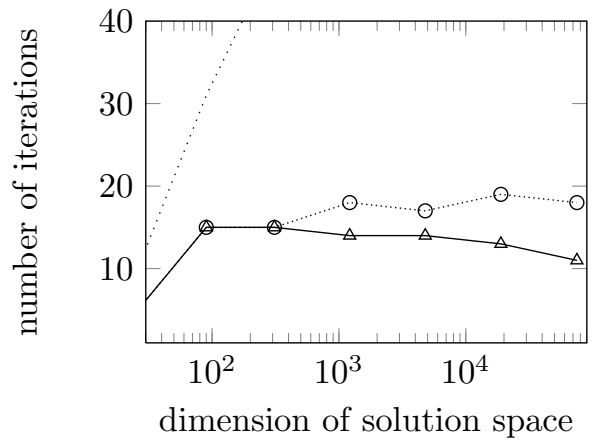

(e) Circle in $\boldsymbol{A}_{z}$

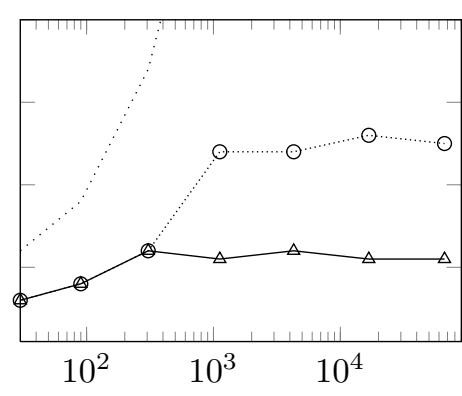

dimension of solution space

(b) Triangle in $\boldsymbol{A}_{\mathrm{d}}$.

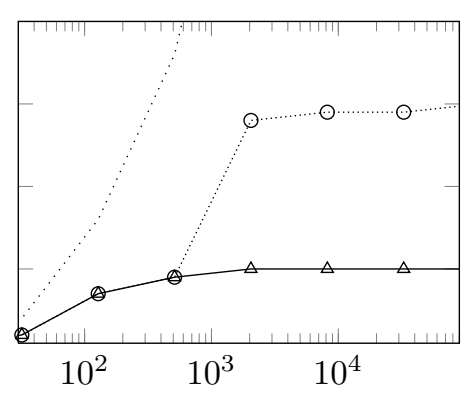

dimension of solution space

(d) Rectangle in $\boldsymbol{A}_{\mathrm{d}}$.

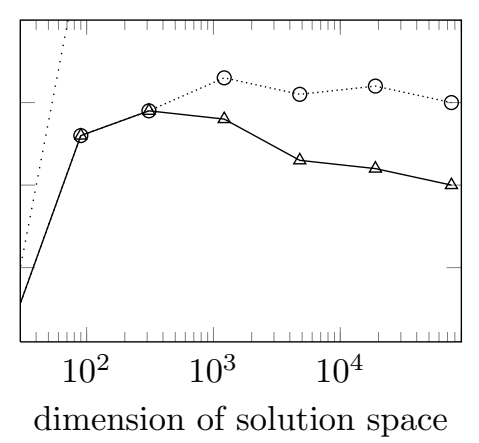

(f) Circle in $\boldsymbol{A}_{\mathrm{d}}$.

Figure 4: MINRES performance for different two-dimensional domains and magnetic vector potentials at the state $\psi_{0} \equiv 1$. The plots show the number of iterations necessary to reach the relative residual of $10^{-11}$ in the norm given by (22) as a function of the dimension of the problem. Starting guess for the linear iterations is $\phi_{0} \equiv 0$ throughout. Plotted are results for the unpreconditioned problem $\ldots . .$. , the preconditioner $R_{1}^{(h)}\left(\psi_{0}^{(h)}\right) \ldots$, and the preconditioner $R_{\infty}^{(h)}\left(\psi_{0}^{(h)} \backsim \triangle\right.$. 


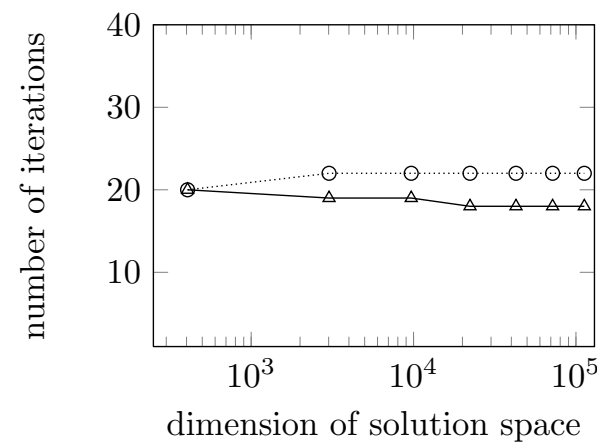

(a) Tetrahedron in $\boldsymbol{A}_{z}$.

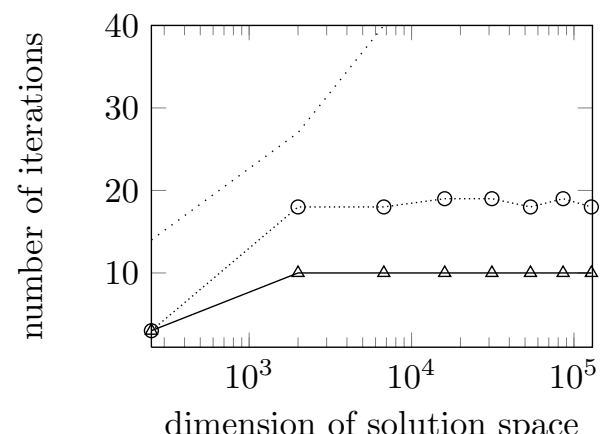

(c) Cube in $\boldsymbol{A}_{z}$.

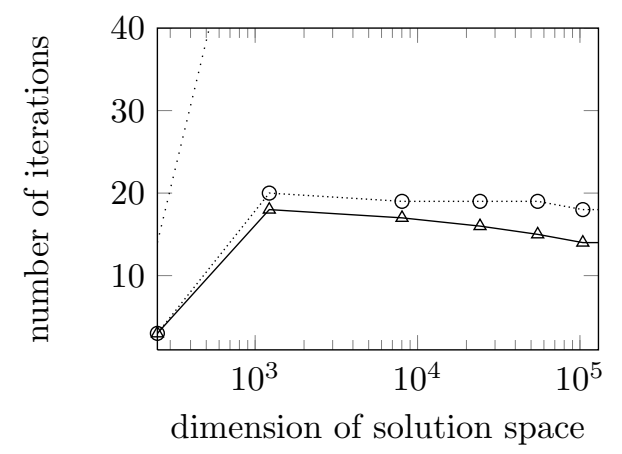

(e) Ball in $\boldsymbol{A}_{z}$.

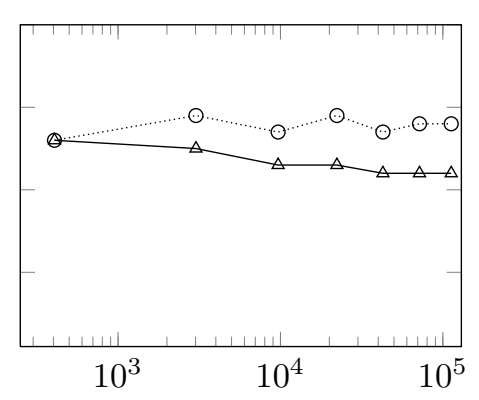

dimension of solution space

(b) Tetrahedron in $\boldsymbol{A}_{\mathrm{d}}$.

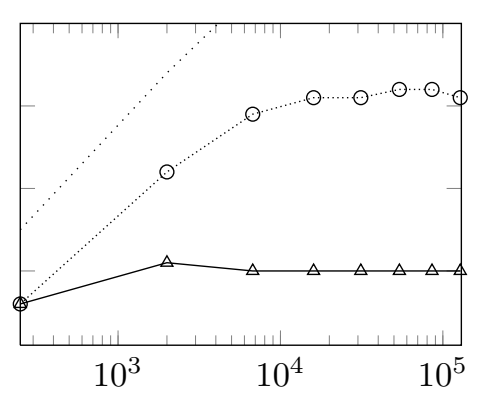

dimension of solution space

(d) Cube in $\boldsymbol{A}_{\mathrm{d}}$.

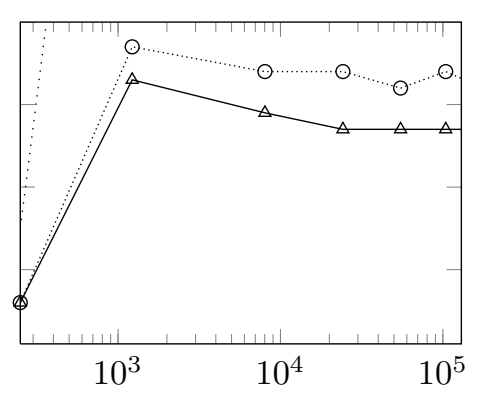

dimension of solution space

(f) Ball in $\boldsymbol{A}_{\mathrm{d}}$.

Figure 5: MINRES performance for different three-dimensional domains and magnetic vector potentials at the state $\psi_{0} \equiv 1$. The plots show the number of iterations necessary to reach the relative residual of $10^{-11}$ in the norm given by (22) as a function of the dimension of the problem. Starting guess for the linear iterations is $\phi_{0} \equiv 0$ throughout. Plotted are results for the unpreconditioned problem $\ldots . .$. , the preconditioner $R_{1}^{(h)}\left(\psi_{0}^{(h)}\right) \ldots \odot$, and the preconditioner $R_{\infty}^{(h)}\left(\psi_{0}^{(h)} \backsim \triangle\right.$. 
the continuation curve, the number of iterations in the preconditioned MINRES solver that was required to solve the Jacobian system up to $\|r\| \leq 10^{-8}$ in the last Newton step. While initially around 50 iterations are required, the introduction of an unstable eigenvalue at the swallow tail $(\mu \approx 0.30)$ slows down MINRES convergence. This is due to the fact that the positive-definite preconditioner does not capture negative eigenvalues. While the convergence is not slowed down by an order of magnitude, the highly unstable high-energy states around $\mu=0.8$ require up to 150 MINRES iterations to converge. The local peaks in the iteration requirements are due to the inherent loss of orthogonality of Krylov basis vectors in MINRES [24]. This effect can be alleviated by storing and fully reorthogonalizing the Krylov basis in each MINRES step such as implemented in GMRES.

\section{Conclusions}

The authors propose a preconditioned Newton-Krylov algorithm that solves the extreme type-II Ginzburg-Landau equation. The solution method uses an AMG preconditioning strategy that yields optimal convergence and scalability for mesoscopic domains.

The Ginzburg-Landau operator consists of a kinetic energy operator that depends on the given magnetic field and a nonlinear reaction term. The linearization of the operator is analyzed and it is found that the Jacobian of the system is self-adjoint with respect to the nonstandard inner product (3). Its spectrum is indefinite if $\psi$ describes a physically unstable solution of the equation. The properties of the kinetic energy operator $K$ are also discussed and it is found to be self-adjoint and positive (semi)-definite. These properties are maintained after discretization with finite volumes and link variables. The proposed preconditioner takes advantage of this by applying an algebraic multigrid scheme to the operator $P^{(h)}\left(\psi^{(h)}\right)=K^{(h)}+2\left|\psi^{(h)}\right|^{2}$. Numerical results for representative domains point towards the optimality of the algorithm in the sense of independence of the number of linear solver iterations from the discretization resolution. This suggests that, qualitatively, no further improvement can be reached.

Moreover, the performance of the preconditioner is assessed in a numerical parameter continuation context where a family of solutions is generated for changing strength of the applied magnetic field. The good convergence results from the test domains are confirmed here. The presence of negative eigenvalues, however, slows down the Krylov convergence if used with a CG solver (figure 6b). Moreover, other factors, such as a large domain size, have shown to hamper the convergence. To gain deeper insight into the convergence behaviors, clearer results than 25 on the spectrum of $\left(P^{(h)}\right)^{-1} J^{(h)}$ are needed.

Nevertheless, this research opens up new possibilities for the exploration of the energy landscape of type-II superconductors. Computation of threedimensional problems are now accessible with grid resolutions on par with current two-dimensional calculations.

A natural extension of the presented work is to approach the solution of the full Ginzburg-Landau problem in which the magnetic vector potential cannot be treated as given [15]. Numerous numerical and computational challenges are posed there, e.g., how to efficiently solve the Jacobian system. The presented preconditioner could be used to construct a block-preconditioning strategy for the general (nonextreme-type-II) Ginzburg-Landau equations. 


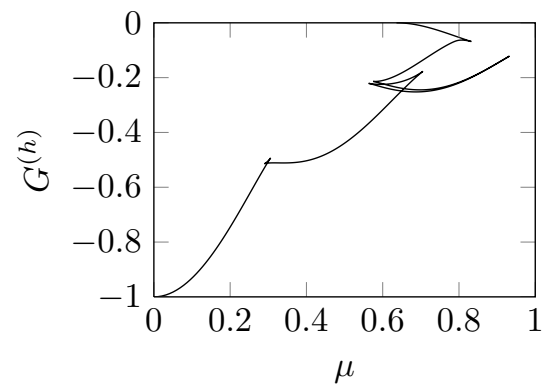

(a) Gibbs energy as a function of $\mu$. Note the so-called swallow tails which are typical for numerical parameter continuation. Structural transitions in the solutions usually happen around here.

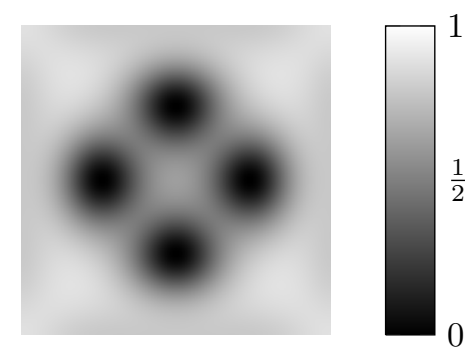

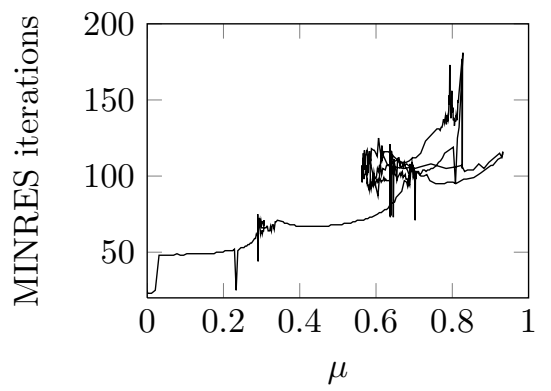

(b) Number of MINRES iterations till $\|r\| \leq$ $10^{-8}$ in the last Newton step as a function of $\mu$. With increasing energy, stability is lost and the presence of unstable eigenmodes hampers the performance of the preconditioner.

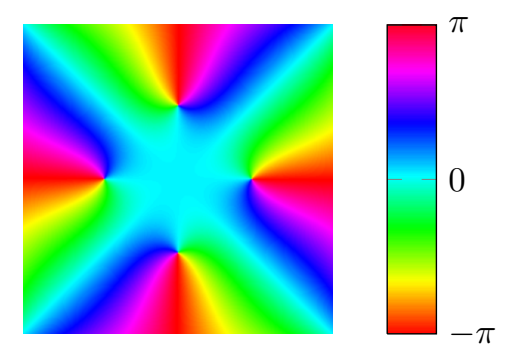

(c) Solution $|\psi|^{2}$, arg $\psi$ at continuation step 22 with $\mu \approx 0.47, F^{(h)} \approx-0.47$. The system has undergone a first transition and four vortices have moved in.
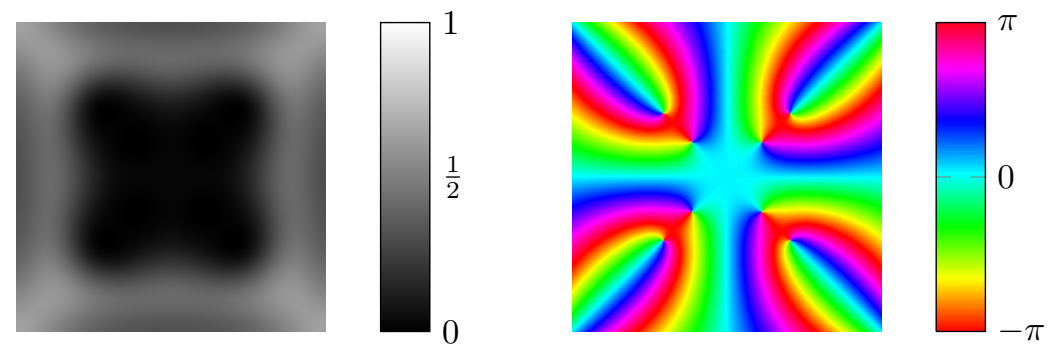

(d) Solution $|\psi|^{2}$, $\arg \psi$ at continuation step 49 with $\mu \approx 0.93, F^{(h)} \approx-0.12$. The system has undergone a second transition and now contains eight vortices.

Figure 6: The performance of the preconditioned Krylov solver in the context of numerical continuation in $\mu \boldsymbol{A}_{z}$ for $\Omega_{\mathrm{sq}}^{(h)}$ with edge length $10,1000^{2}$ unknowns. The solution is continued in the parameter $\mu$ with the help of pseudo arc-length continuation where each continuation step requires the solution of a nonlinear system. 


\section{Acknowledgments}

We acknowledge fruitful discussions with Qiang Du, Andrew G. Salinger, Gregory D. Sjaardema, and Mark Hoemmen. We are also grateful to the Research Foundation Flanders (FWO) for financial support through the project G017408N.

\section{References}

[1] J. Taylor, Optical solitons: Theory and experiment, Vol. 10, Cambridge University Press., 1992.

[2] W. Bao, D. Jaksch, P. A. Markowich, Numerical solution of the Gross-Pitaevskii equation for Bose-Einstein condensation, Journal of Computational Physics 187 (1) (2003) 318-342. doi:10.1016/S0021-9991(03)00102-5.

URL http://www.sciencedirect.com/science/article/pii/ S0021999103001025

[3] A. Abrikosov, Magnetic properties of superconductors of the second group, Sov. Phys. JETP 5 (1957) 1174.

[4] E. Sandier, S. Serfaty, Vortices in the magnetic Ginzburg-Landau model, Birkhäuser, 2007.

[5] F.-H. Lin, Q. Du, Ginzburg-Landau vortices: Dynamics, pinning, and hysteresis, SIAM J. Math. Anal. 28 (6) (1997) 1265-1293. doi:http: //dx.doi.org/10.1137/S0036141096298060.

[6] Q. Du, M. D. Gunzburger, J. S. Peterson, Modeling and analysis of a periodic Ginzburg-Landau model for type-II superconductors, SIAM J. Appl. Math. 53 (3) (1993) 689-717. doi:http://dx.doi.org/10.1137/0153035

[7] V. Schweigert, F. Peeters, P. Singha Deo, Vortex phase diagram for mesoscopic superconducting disks, Phys. Rev. Lett. 81 (1998) 2783-2786. doi:10.1103/PhysRevLett.81.2783.

URL http://link.aps.org/doi/10.1103/PhysRevLett.81.2783

[8] M. Milošević, F. Peeters, Superconducting Wigner vortex molecule near a magnetic disk, Phys. Rev. B 68 (2003) 024509. doi:10.1103/PhysRevB 68.024509.

URL http://link.aps.org/doi/10.1103/PhysRevB.68.024509

[9] D. Knoll, D. Keyes, Jacobian-free Newton-Krylov methods: A survey of approaches and applications, Journal of Computational Physics 193 (2) (2004) 357-397.

[10] C. Yang, X. Cai, D. E. Keyes, M. Pernice, Parallel domain decomposition methods for the 3D Cahn-Hilliard equation, Proceedings of the 2011 annual meeting of DOE's Scientific Discovery through Advanced Computing.

[11] G. F. Carey, D. J. Knezevic, Multiscale and hysteresis effects in vortex pattern simulations for Ginzburg-Landau problems, International Journal for Numerical Methods in Engineering 81 (7) (2010) 866-891. doi:10.1002/ nme.2714

URL http://dx.doi.org/10.1002/nme.2714 
[12] B. Krauskopf, Numerical Continuation Methods for Dynamical Systems: Path following and boundary value problems, Springer Verlag, 2007.

[13] N. Schlömer, D. Avitabile, W. Vanroose, Numerical bifurcation study of superconducting patterns on a square, SIAM J. Appl. Dyn. Syst. 11 (2012) $447-477$.

[14] R. Huebener, Magnetic flux structures in superconductors, Vol. 6, Springer Verlag, 2001.

[15] Q. Du, M. D. Gunzburger, J. S. Peterson, Analysis and approximation of the Ginzburg-Landau model of superconductivity, SIAM Rev. 34 (1992) $54-81$.

[16] H. Kaper, M. Kwong, Vortex configurations in type-II superconducting films, Journal of Computational Physics 119 (1) (1995) 120-131.

[17] Q. Du, Numerical approximations of the Ginzburg-Landau models for superconductivity, Journal of mathematical physics 46 (2005) 095109.

[18] H. Van der Vorst, Iterative Krylov methods for large linear systems, Vol. 13, Cambridge University Press, 2003.

[19] J. B. Schroder, Smoothed aggregation solvers for anisotropic diffusion, Numerical Linear Algebra with Applications 19 (2012) 296312.

[20] N. Bell, L. Olson, Algebraic multigrid for $k$-form Laplacians, Numerical Linear Algebra with Applications 15 (2-3) (2008) 165-185.

[21] M. Gee, C. Siefert, J. Hu, R. Tuminaro, M. Sala, ML 5.0 smoothed aggregation user's guide, Tech. Rep. SAND2006-2649, Sandia National Laboratories (2006).

[22] S. P. MacLachlan, C. W. Oosterlee, Algebraic multigrid solvers for complexvalued matrices, SIAM Journal on Scientific Computing 30 (3) (2008) 1548-1571. doi:10.1137/070687232.

URL http://link. aip.org/link/?SCE/30/1548/1

[23] W. Bell, L. Olson, J. Schroder, PyAMG: Algebraic multigrid solvers in Python, version 1.1 (2008).

URL http://www.pyamg.org

[24] G. L. G. Sleijpen, H. A. van der Vorst, J. Modersitzki, Differences in the effects of rounding errors in Krylov solvers for symmetric indefinite linear systems, SIAM J. Matrix Anal. Appl. 22 (3) (2000) 726-751 (electronic). doi: $10.1137 /$ S0895479897323087.

URL http://dx.doi.org/10.1137/S0895479897323087 